\title{
Plasticidade Neural, um caminho para a aprendizagem: breve análise
}

\author{
Neural plasticity, a path to learning: a brief analysis \\ Plasticidad neuronal, un camino hacia el aprendizaje: un breve análisis
}

\section{Resumo}

A educação relaciona-se com outras áreas de saber, assim como com a neurociência. Por intermédio da descoberta dessa e da plasticidade cerebral ou neural, pode-se revelar a complexidade da aprendizagem a nível biológico, assim como auxiliar o desenvolvimento de novas metodologias e formas de aprendizagem. Nesse contexto, o estudo visou analisar as relações entre plasticidade cerebral e aprendizagem, tendo a neurociência como ponto de partida. Para tal, utilizou-se de pesquisa bibliográfica, feita em meio virtual e material, na qual foram pontuados os resultados e tecidos os diálogos entre as fontes, sendo esses expressos em dois tópicos em resultados e discussão. As conclusões e impressões da autora estão nas considerações finais.

Palavras-chave: Neurociência; Plasticidade neural; Aprendizagem.

\begin{abstract}
Education is related to other areas of knowledge, as well as neuroscience. Through the discovery of this and brain or neural plasticity, the complexity of learning at a biological level can be revealed, as well as helping the development of new methodologies and forms of learning. In this context, the study aimed to analyze the relationships between brain plasticity and learning, having neuroscience as a starting point. To this end, a bibliographic research was used, carried out in a virtual and material medium, in which the results were scored and the dialogues between the sources were scored, which were expressed in two topics in results and discussion. The author's conclusions and impressions are in the final considerations.
\end{abstract}

Keywords: Neuroscience; Neural plasticity; Learning.

\section{Resumen}

La educación está relacionada con otras áreas del conocimiento, así como con la neurociencia. A través del descubrimiento de esta y de la plasticidad cerebral o neuronal, se puede revelar la complejidad del aprendizaje a nivel biológico, así como ayudar al desarrollo de nuevas metodologías y formas de aprendizaje. En este contexto, el estudio tuvo como objetivo analizar las relaciones entre plasticidad cerebral y aprendizaje, teniendo como punto de partida la neurociencia. Para ello, se utilizó una investigación bibliográfica, realizada en un medio virtual y material, en la que se puntuaron los resultados y se puntuaron los diálogos entre las fuentes, que se expresaron en dos temas en resultados y discusión. Las conclusiones e impresiones del autor están en las consideraciones finales.

Palabras clave: Neurociência; Plasticidad neuronal; Aprendiendo. 


\section{Introdução}

A Educação é uma fonte inesgotável de discussões e debates. Quando o educador observa uma criança respondendo corretamente uma atividade lúdica, por exemplo, até algumas décadas atrás, esse não reconheceria toda a atividade cerebral existente para que essa resposta de aprendizagem aconteça.

A aprendizagem é um processo complexo que está sendo estudado e pesquisado pelas ciências, incluindo a neurociência, que estuda, dentre outras temáticas, a plasticidade cerebral.

Diante da descoberta da plasticidade cerebral e como essa acontece, descobriu-se também como a aprendizagem acontece a nível biológico.

Nesse sentido, o presente estudo visou analisar as relações entre plasticidade cerebral e aprendizagem, tendo a neurociência como ponto de partida.

\section{Metodologia}

A construção deste trabalho foi elaborada através de pesquisa bibliográfica, que segundo Lakatos e Marconi (2010),

[...] abrange toda bibliografia já tornada pública em relação ao tema de estudo, desde publicações avulsas, boletins, jornais, revistas, livros, pesquisas, monografias, teses, material cartográfico etc. [...] Dessa forma, a pesquisa bibliográfica não é mera repetição do que já foi dito ou escrito sobre certo assunto, mas propicia o exame de um tema sob novo enfoque ou abordagem, chegando a conclusões inovadoras (Lakatos, Marconi, 2010, p.166).

O primeiro momento foi o de pesquisa virtual em periódicos científicos na área da Educação e em sites confiáveis, como a base de dados Google Scholar, Scielo, entre outras, utilizando os descritores educação, plasticidade neural e aprendizagem. Foram separados periódicos que continham a temática, porém correlacionadas. Foi feita uma leitura analítica, pontuando as convergências e relacionando os textos.

O segundo momento foi de pesquisa em material impresso, como livros e dicionários. Foi feita uma leitura rápida para inclusão e exclusão e após, a leitura analítica tal qual no material virtual.

Um terceiro momento foi o apontamento de reflexões e pontos a serem discutidos, que gerou a discussão do presente trabalho, numa perspectiva de relacionar plasticidade cerebral e aprendizagem.

O quarto momento compreende o desenvolvimento das conclusões juntamente com as impressões da autora, descritas no tópico considerações finais.

\section{Resultados e Discussão}

\subsection{Neurociência e a plasticidade cerebral}

O homem é dotado de capacidades cognitivas inatas a sua condição de ser humano, sendo constante objeto de estudo por arte da ciência, principalmente no que concerne ao sistema neurológico e seus desdobramentos para a saúde e bem-estar, assim como para os processos cógnitos e de aprendizagem.

Segundo Ferrari et al. (2001), as interações que o indivíduo vive entre seu organismo e o ambiente determinam quais respostas serão dadas e quais as funções dessas. As decorrentes relações entre eventos externos e respostas do organismo criam relações de condição entre os eventos externos e as respostas, que geram mecanismos de sobrevivência e adaptabilidade. Esses são fatores determinantes para que ocorram mudanças na forma, tamanho e funções do sistema nervoso (Bussab, 2000; Catania, 1999; Skinner, 1981 apud Ferrari et al., 2001). 
Como resultado da evolução, o cérebro possui abundância de sistemas neurais que são transformados pelas experiências. Esta capacidade de transformação denota a plasticidade cerebral, que está presente no ser humano durante todas as fases de sua vida (Ferrari et al., 2001).

Atualmente, a característica que predomina no sistema nervoso é a plasticidade constante que esse possui (Lima, 2020). Cosenza e Guerra (2011 apud Lima, 2020) corroboram que a plasticidade neural não é algo específico dos primeiros anos de vida, mas que permanece para toda a vida dado que essa noção de mudança estrutural induzida existe desde os estudos de Santiago Ramon y Cajal (Freire MAM. Santiago Ramon y Cajal - O pai da neurociência moderna. Neurociências 2006; 3 : 146-147). Nesse contexto, as sinapses são regiões onde ocorrem a passagem de informações entre os neurônios, feitas e desfeitas de acordo com as interações constantes entre o ambiente interno e externo. Segundo os mesmos, no decorrer do desenvolvimento da plasticidade, entre oportunidades, são adquiridas competências e habilidades, como os exemplos: visão (do nascimento até os 6 anos), controle emocional (9 meses aos 6 anos), linguagem (dos 9 meses aos 8 anos), habilidades sociais (dos 4 anos aos 8 anos), música (dos 4 anos aos 11 anos), segundo idioma (dos 18 meses aos 11 anos).

A ciência que estuda esses processos, integrando diversas áreas de saber como metodologias, neurofisiologias, psicologia, farmácia, bioquímica, anatomia e genética é chamada de neurociência, que tem como princípio fundamental que a atividade das células neurais são determinadas pelos ambientes físicos e sociais (Ferrari, 2001).

De acordo com Pia (1985 apud Ferrari et al., 2001), o termo plasticidade surgiu na literatura, em 1930, introduzido pelo fisiologista alemão Albrecht Bethe, sendo considerada como a capacidade de adaptação a mudanças no ambiente tanto externas como internas, devido à sinergia entre os órgãos, coordenada pelo Sistema Nervoso Central (SNC).

As pesquisas que abordam essa temática, seguindo os determinantes de Kolb \& Whishaw (1989 apud Ferrari et al., 2001, s. p.), são classificadas em três tipos:

(a) metabólicas: que analisam alterações da atividade metabólica em áreas corticais e subcorticais, tanto no mesmo hemisfério em que se localizam as lesões (ipsilaterais) quanto no hemisfério oposto (contralaterais);

(b) neuroquímicas: que focalizam as alterações funcionais nas sinapses, investigando processos/mecanismos que aumentam a síntese de neurotransmissores, a liberação de neurotransmissores ou a potencialização das respostas póssinápticas, em decorrência de situações estimuladoras, de aprendizagem ou de lesões;

(c) morfológicas: que caracterizam e enfatizam as modificações na estrutura das sinapses e neurônios, tais como a regeneração e ramificação de axônios, aumento do tamanho de corpos celulares, do número de dendritos, do número de neurônios e de sinapses. Essas categorias não são exclusivas e podem ser combinadas em um mesmo estudo.

Amauri Betini Bartoszeck (2009, p. 1 apud Lima, 2020, p.31) corroboram que a neurociência é uma disciplina recente que compreende a neurologia, a psicologia e a biologia, e que diante de novas descobertas, estudos e pesquisas, está ocorrendo a união de áreas de saberes afins, como o caso da biologia e pedagogia, para a compreensão de atividades e estruturas cerebrais durante o processo de aprendizagem, o que tem fomentado discussões acerca de novas metodologias que envolvem estratégias relacionadas com questões neurais (Bortoli; Teruya, 2017).

\subsection{Plasticidade cerebral e aprendizagem}

A aprendizagem permanece sendo temática de muitas discussões no âmbito educacional. A cada nova descoberta, novos conceitos, significados e relações são estabelecidos, principalmente quando se trata de neurociência, plasticidade cerebral e cérebro (centro das atividades de maior complexidade, o pensamento, a memória e as emoções) (Aamodt \& Wang, 2013 apud Bortoli \& Teruya, 2017).

Na perspectiva da neurociência, a aprendizagem compreende alterações no cérebro resultantes de experiências vividas (Sant'Ana, 2015 apud Bortoli \& Teruya, 2017); processo em que são adquiridas informações (Gazanniga, 2006, p. 320), sendo 
reconhecido como plasticidade neural "reorganizações de funções e estruturas cerebrais, localizadas, portanto, no maior órgão do sistema nervoso, o cérebro" (Sant'Anna, 2015, p. 73 apud Bortoli; Teruya, 2017, p. 74).

Corroboram Cosenza e Guerra (2011 apud Lima, 2020) que algumas experiências realizadas com animais apresentaram que algumas habilidades e competências se desenvolvem de maneira plena mediante estímulos, ou seja, existem períodos críticos, nos quais o desenvolvimento acontece corretamente. Porém, em humanos, ainda podem ocorrer em outras épocas pois a aprendizagem é um processo contínuo, todavia necessitando de muito mais trabalho para que ocorra.

De acordo com Relvas (2015), a plasticidade ocorre de três maneiras distintas: no desenvolvimento de cérebro normal, em resposta a uma experiência e em reação a uma lesão na tentativa de reorganizar o Sistema Nervoso Central, porém o autor destaca que para fins de estudo da aprendizagem, deve ser utilizada a maneira de desenvolvimento de cérebro norma. Nesse sentido a aprendizagem acontece mediante estímulos constantes, e sua eficácia por intermédio do ambiente onde o indivíduo está inserido (Cosenza; Guerra, 2011 apud Lima, 2020).

Sant'ana (2015) afirma que a aprendizagem, que provoca mudanças no comportamento, está estreitamente relacionada às mudanças biológicas, provocadas por desafios motivadores constantes (novas estruturas cerebrais). A Figura 1 expressa a correlação:

Figura 1: Inter-relação entre plasticidade neural e aprendizado.

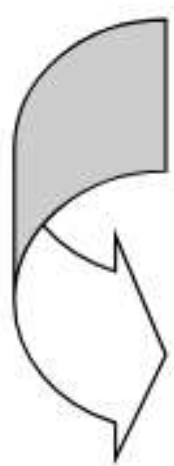

\section{PLASTICIDADE NEURAL}

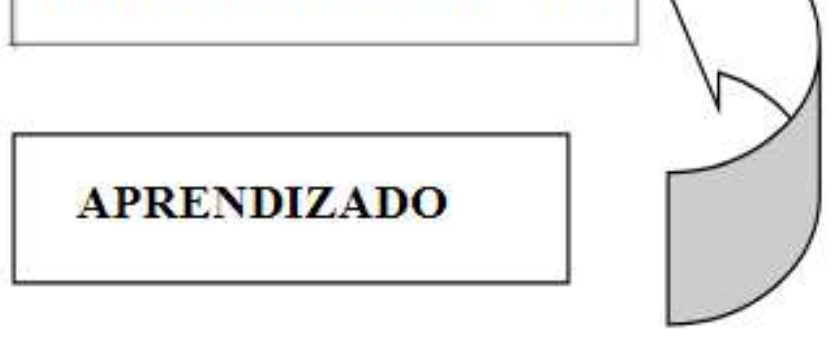

Fonte: Sant'ana (2015, p. 77 apud Lima, 2020).

Destacando a importância de fatores externos para a plasticidade neural e consequente aprendizagem, Sant'ana (2015) expõe alguns fatores mais comuns percebidos em suas pesquisas, que são: hormônios, medicamentos, sono, alimentação, genética, drogas, doenças, estímulos. Destaca-se o fato de que existem fatores interventores positivos e negativos.

A Figura 2 expressa essa dinâmica. 
Figura 2: Fatores do ambiente e plasticidade neural.

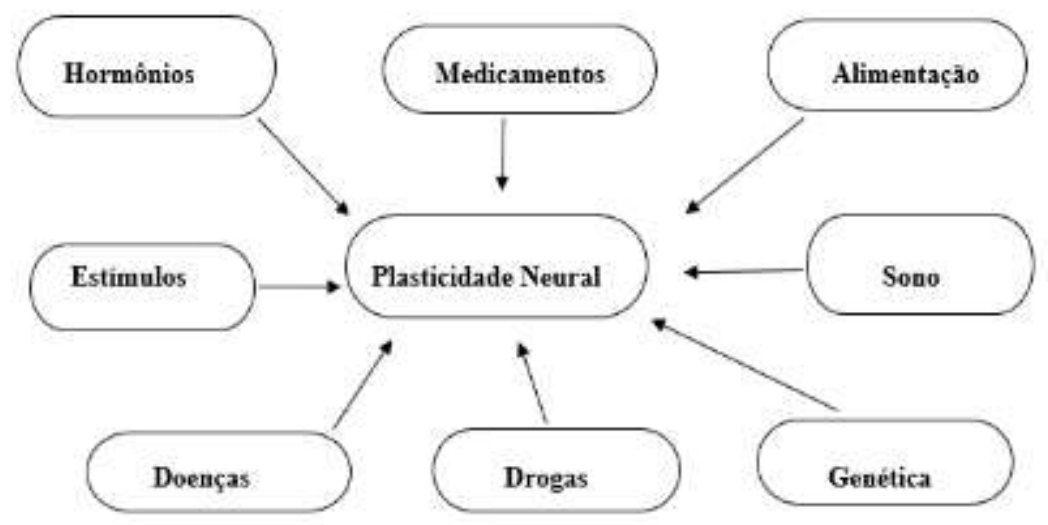

Fonte: Sant'Ana (2015, p. 77 apud Lima, 2020).

Lima (2020) destaca o fato de muitos serem os envolvidos no desenvolvimento tanto da plasticidade neural como da aprendizagem, oferecendo destaque para pais, familiares, escola e principalmente os professores quando o sujeito está em idade escolar, pois esse torna-se um fator determinante no processo de aquisição de aprendizagem.

Nessa perspectiva, destaca-se a necessidade de releituras a respeito de práticas pedagógicas, acrescentando relevantes descobertas da neurociência sobre a plasticidade neural.

Portanto, as descobertas advindas através de estudos e pesquisas de neurociência a aproximam da Educação, confirmando que a aprendizagem é um processo biológico que modifica a plasticidade cerebral, gerando uma relação de reciprocidade (interrelação).

\section{Considerações Finais}

A neurociência como ciência que se destina a ser, muito tem colaborado para o desenvolvimento de metodologias, ferramentas e instrumentos utilizados por educadores para facilitar, intensificar e melhorar o processo de aprendizagem, que é complexo.

A descoberta da aprendizagem a nível biológico e a plasticidade neural trouxeram consigo inúmeros esclarecimentos e novas leituras a respeito da educação praticada anteriormente.

Portanto, a neurociência é uma eficiente aliada do processo educativo, no que concerne a aquisição de aprendizagens.

\section{Referências}

Aldskogius, H. \& Kozlova, E. (1998). Central neuron-glial and glial-glial interactions following axon injury. Progress in Neurobiology, 55, 1-26.

Bortoli, B. de, \& Teruya, T. K. Neurociência e educação: os percalços e possibilidades de um caminho em construção. Imagens da Educação, 7(1),70-77, 2017. https://periodicos.uem.br/ojs/index.php/ImagensEduc/article/view/32171/pdf.

Bzuneck, J. A. Emoções acadêmicas, autorregulação e seu impacto sobre motivação e aprendizagem. ETD-Educação Temática Digital, 20(4), 1059-1075, 2018. https://periodicos.sbu.unicamp.br/ojs/index.php/etd/article/view/8650251/1867>.

Cerutti, S. M. \& Ferrari, E. A. M. (1995a). Funções da neuroglia no sistema nervoso central: contribuições para a plasticidade neural. LECTA: Revista de Farmácia e Biologia, 13, 79-100.

Da Silva, M. do C. G., et al. Plasticidade neural, neurociência e educação: as bases do aprendizado. Arquivos do Mudi, 24(2), 30-41, 2020. https://periodicos.uem.br/ojs/index.php/ArqMudi/article/view/53548/751375150705>.

Ferrari, E. A. et al. Plasticidade neural: relações com o comportamento e abordagens experimentais. Psicologia: teoria e pesquisa, 17, 187-194, https://www.scielo.br/j/ptp/a/ysvrdSJm8fSR5fTsdYjMFXM/?lang=pt>. 
Research, Society and Development, v. 10, n. 16, e553101624103, 2021

(CC BY 4.0) | ISSN 2525-3409 | DOI: http://dx.doi.org/10.33448/rsd-v10i16.24103

Fonseca, V. da. Importância das emoções na aprendizagem: uma abordagem neuropsicopedagógica. Revista Psicopedagogia, 33(102), 365-384, 2016.

Lakatos. E. M., \& Marconi, M. de A. Fundamentos de Metodologia Científica. (7a ed.), Atlas, 2010.

Lima, E. S. Cérebro Humano e educação hoje. In: Revista Presença Pedagógica 16(94). https://periodicos.uem.br/ojs/index.php/ArqMudi/article/view/53548/751375150705>.

Moraes, S., \& M. F. de M. M. "Psicomotricidade no contexto da neuroaprendizagem: contribuições à ação psicopedagógica." Revista Psicopedagogia 32.97 (2015): 84-92.

Morales, M. N. Experiencia de Aprendizaje Mediado. Aula proferida na Universidad del Mar no programa de doutorado. Chile janeiro de 2010.

Seixas, S. R. P. M. M. "Da neurobiologia das relações precoces à neuroeducação." Interacções 30.10 (2014): 44-71.

Skinner, B. F. (1981). Selection by consequences. Science, 213, 501-

504.

Stein, D. G., Finger, S. \& Hart, T. (1983). Brain damage and recovery: Problems and perspectives. Behavioural and Neural Biology, $37,185-222$.

Strumwasser, F. (1994). The relations between neuroscience and human behavioral science. Journal of the Experimental Analysis of Behavior, $61,307-317$.

Tsukahara, N. (1981). Synaptic plasticity in the mammalian central nervous system. Annual Review of Neuroscience, 4, 351-379.

Weinberger, N. M. \& Diamond, D. M. (1987). Physiological plasticity in auditory cortex: Rapid induction by learning. Progress in Neurobiology, 29, 1-5.

Welker, E., Rao, S.B., Dorfl, J., Melzer, P. \& Van Der Loos, H. (1991). Plasticity in the barrel cortex of the adult mouse: Effects of chronic stimulation upon deoxyglucose uptake in the behaving animal. Journal of Neuroscience, 12, 153-170

Vygotsky. L. A Formação Social da Mente o desenvolvimento dos processos psicológicos superiores. Martins Fontes, 1998.

Rolim, A. A. M., Siena., S. F. G., \& Mônica, M. T. "Uma leitura de Vygotsky sobre o brincar na aprendizagem e no desenvolvimento infantil." Revista Humanidades 23.2 (2008): 176-180 